\title{
Educación para el emprendimiento: una propuesta para el desarrollo humano*
}

\section{Entrepreneurship education: a proposal for human development}

\author{
Dra. Arantxa AZQUETA. Profesora Asociada. Universidad de Navarra (aazqueta@unav.es). \\ Dra. Concepción NAVAL. Catedrática. Universidad de Navarra (cnava(@unav.es).
}

\section{Resumen:}

El interés por la educación emprendedora se presenta como una tendencia educativa internacional que ha calado con fuerza en la institución educativa. Se promueve su desarrollo en todas las etapas educativas, desde la educación temprana a la educación superior. Esta aportación analiza la inclusión de la educación para el emprendimiento en el currículo desde una perspectiva crítica, profundizando en la búsqueda de las claves que permitan fortalecer el valor educativo de la educación emprendedora, desde los planteamientos de la antropología humanista. Para ello, se emplea una metodología de investigación teórica con un enfoque analítico e interpretativo, fundamentado en la literatura académica y se busca la reflexión que favorezca, con posterioridad, el desarrollo de líneas de mejora en la práctica educativa. La educación emprendedora se define como el enfoque educativo que posibilita el crecimiento del potencial emprendedor de los estudiantes y contribuye al crecimiento integral, principalmente de las dimensiones intelectual, societaria y moral de la persona, y no se limita al crecimiento socioeconómico y laboral. Favorece, en primer lugar, el crecimiento de la inteligencia a través del desarrollo de la creatividad y de la innovación, anclado en el carácter irrepetible del ser humano y de su libertad; en segundo lugar, el crecimiento social, que promueve la relacionalidad más allá de la reciprocidad, busca el bien común y no solo el bienestar económico, y favorece el desarrollo de virtudes sociales como la solidaridad y la

\footnotetext{
* Este trabajo se enmarca dentro de la producción investigadora del grupo de investigación consolidado de la Universidad de Navarra: Educación, Ciudadanía y Carácter (https://www.unav.edu/web/facultad-de-educacion-ypsicologia/educacion-y-ciudadania).
}

Fecha de recepción de la versión definitiva de este artículo: 22-11-2018.

Cómo citar este artículo: Azqueta, A. y Naval, C. (2019). Educación para el emprendimiento: una propuesta para el desarrollo humano | Entrepreneurship education: a proposal for human development. Revista Española de Pedagogía, 77 (274), 517-533. doi: https://doi.org/10.22550/REP77-3-2019-03 
cooperación; y para finalizar, el crecimiento moral, que supone el desarrollo de la libertad e implica el crecimiento de la autonomía y el liderazgo. Esta propuesta de educación para el emprendimiento se inscribe en una línea de trabajo que subraya la finalidad intrínseca de la educación, pone en primer lugar las necesidades personales del educando y posibilita que la educación emprendedora manifieste la relación explícita entre educación, persona y sociedad.

Descriptores: educación emprendedora, antropología educativa, educación humanística, educación y desarrollo.

\section{Abstract:}

\section{Introducción}

En los últimos años, el interés por una cultura emprendedora ha adquirido gran fuerza en nuestra sociedad. Se considera un medio que propicia el cambio de mentalidad que necesita la sociedad europea para adaptarse a los requerimientos del siglo XXI ening the value of entrepreneurship education using a humanistic anthropological paradigm. Entrepreneurship education is defined as an educational focus that enhances entrepreneurial potential in students and contributes to their all-round growth. It is not just limited to socio-economic and professional growth but places a particular focus on the intellectual, social, and moral dimensions of their development. First of all, it helps to stimulate intelligence by developing creativity and innovation, both of which are intrinsic and unique to all human beings and to their freedom. Secondly, it promotes social development, encouraging relationships that transcend mere reciprocity and aim to find the common good and not just economic well-being. Finally, it stimulates moral development, which involves an awareness of freedom and entails autonomy and leadership. This proposal for entrepreneurship education is part of a line of work that underlines the intrinsic goal of education by focusing on the personal needs of the student and allows for entrepreneurial development to manifest the explicit relationship between the individual, education, and society.

Keywords: entrepreneurship education, educational anthropology, humanistic education, educational development.
(Asamblea General de las Naciones Unidas, 2000, 2015; Consejo Europeo, 2000; Comisión Europea, 2012, 2014). Esta situación ha aumentado la atención por la educación emprendedora en todo el mundo (Nabi, Liñán, Fayolle, Krueger y Walmsley, 2017; Hornsby, Messersmith, Rutherford y Simmons, 2018). 
En el ámbito español, la educación del talento emprendedor se ha convertido en la última década en un propósito de nuestro sistema educativo. Tanto la LOE (2/2006) como la LOMCE (8/2013) señalan, en sus preámbulos, su compromiso decidido con los objetivos e intereses planteados por Naciones Unidas y la Unión Europea desde el inicio del siglo xxI. La Ley 14/2013, de apoyo a los emprendedores y su internacionalización, menciona la necesidad de reforzar la cultura emprendedora a través de la educación. Asimismo, conviene resaltar que la inscripción de la competencia sentido de iniciativa y espíritu emprendedor como claves dentro de la educación obligatoria imprime a esta competencia carácter de perdurabilidad (Orden ECD 65/2015).

Esta situación ha supuesto el fortalecimiento de los lazos entre las instituciones de educación y el interés por el emprendimiento. Se plantea como deseable que la educación emprendedora ocupe un lugar en las aulas. Este contexto incentiva a los investigadores en educación a aportar una fundamentación teórica, tarea en la que desea colaborar el presente artículo. El objetivo principal de este trabajo es favorecer la reflexión y facilitar el desarrollo de líneas de mejora en la práctica educativa. La metodología de investigación es la propia de una investigación teórica que sigue un enfoque de estudio analítico e interpretativo fundamentado en la literatura académica. Se ha elegido este método porque se considera el apropiado para un trabajo de tipo conceptual y teórico en la que se busca comprender el fenómeno emprendedor y aportar información de interés para el ámbito educativo.

\section{La inclusión de la formación para el emprendimiento en la educación: análisis crítico}

Existe una abundante bibliografía sobre educación para el emprendimiento. Esto confirma el interés por esta disciplina desde muy variadas perspectivas, aunque prevalece el enfoque que lo asocia con el crecimiento económico y la generación de empresas (Jones, Maas y Pittaway, 2017; Bernal Guerrero y Cárdenas, 2017; Nabi et al., 2017). Nos encontramos en una época de profundas modificaciones en el sector económico, fruto de la revolución tecnológica y la globalización en la que se gesta un modelo económico en el que priman los valores emprendedores.

En este modelo, el conocimiento, prioritariamente técnico y científico, es el factor dominante de la producción. La creatividad, la capacidad de comunicarse y la inteligencia emocional son factores importantes y la innovación es una ventaja competitiva. Tener capacidad de innovar y de asumir riesgos es pieza clave en la sociedad del conocimiento (Bernal Guerrero, Granados y González Donoso, 2014).

La inversión en educación, a medio y largo plazo, reactiva las economías y equilibra los sistemas industriales, que carecen de personal cualificado y demandan trabajadores con habilidades específicas que aporten valor en el mercado. La innovación exitosa depende de la creatividad humana -conocimientos, habilidades y talentos- que se desarrollan, en gran parte, a través de la educación. De esta manera, el sistema educativo común europeo, con la formación basada en competencias 
vincula las demandas sociales y laborales con las necesidades del mercado (Michavila, Martínez, Martín-González, García-Peñalvo y Cruz-Benito, 2016; Martínez Clares y González Morga, 2018).

Los esfuerzos que impulsan las reformas educativas actuales tienen principalmente una inspiración neoliberal que marca la política educativa impulsada desde la Unión Europea (Erkkilä, 2000; Rae, 2010; Komulainen, Naskali, Korhonen y Keskitalo-Foley, 2011; Business, Innovation \& Skills Department, 2015; Fougère, Segercrantz y Seeck, 2017). El razonamiento que sustenta esta concepción educativa, en la que nos queremos centrar, es la teoría del capital humano esbozada y consolidada a mediados del siglo pasado a partir del estudio sociológico realizado por Theodore Schultz (1961); Gary Becker (1964) y Jacob Mincer (1974). Esta teoría se complementa con la perspectiva de la sociología funcionalista de la realidad, que se caracteriza por el utilitarismo y concede la máxima importancia a la innovación, la creación tecnológica y el interés por la capacidad económica de los individuos y de la sociedad (Espejo, 2001). A su vez el emprendimiento sintetiza algunas de las características del capitalismo cognitivo que convierte al emprendedor en un perfil profesional que se adapta a las nuevas prácticas de flexibilidad, iniciativa, creatividad e innovación que requiere el mercado (Ruiz-Herrero, 2013).

Conviene resaltar la necesidad de sustraer del debate político y económico el interés por la educación para el emprendimiento. La educación no está dotada únicamente de un carácter medial para conseguir un fin, sino que es un proyecto en sí misma. Se define en favor del ser humano, y contribuye a su desarrollo personal, no solo al incremento de la productividad y de la rentabilidad económica. Siguiendo a Ibáñez-Martín y Fuentes (2017) el aumento del poder económico no produce una mejora del desarrollo humano sino que se requieren otros condicionantes para que estas capacidades alcancen su culmen, se desarrollen y reviertan a la sociedad. Se requiere fundamentar la educación para el emprendimiento para evitar que su impulso se limite a desarrollar las habilidades que mejoren la capacidad productiva.

Los responsables de las políticas educativas citan el progreso económico como objetivo educativo, y confían en ideas y conceptos económicos que guían su trabajo. En la economía, el progreso se mide a través de la satisfacción de las preferencias; así la mejora del bienestar es su objetivo fundamental. A mayor grado de satisfacción se obtiene mayor bienestar. De este modo, la satisfacción de las preferencias se convierte en objeto de debate para los filósofos de la educación (Gilead, 2012).

Este planteamiento de raíz utilitarista puede convertir a las personas en medios para alcanzar el progreso, en lugar de fines a los que atender y posibilitar un progreso económico. Se puede decir que deshumaniza, porque se trata a la persona como un qué y no como un quién. El fin que preside la acción educativa no puede estar solo dotado de carácter subjetivo, porque entonces solo resolverá una necesidad 
transitoria como es, por ejemplo, aumentar el empleo por cuenta propia, hacer crecer el tejido empresarial o paliar un problema social. Desde este punto de vista, se dota a la educación para el emprendimiento de una funcionalidad cortoplacista, con una motivación extrínseca, encaminada al logro de unos resultados que reclama la sociedad. En cambio, conviene que el interés por la educación para el emprendimiento contribuya al crecimiento y desarrollo integral de la persona humana.

Es preciso no legitimar como materia de interés educativo lo que simplemente goza de racionalidad instrumental o contribuye al progreso de la ciencia y de la tecnología (Bauman, 2013). Una sociedad y una educación excesivamente orientada a la eficiencia y a la utilidad, que limita lo razonable a lo técnico e instrumental, pueden correr el riesgo de deshumanizarse 0 desnaturalizarse. En palabras de Barrio (2013), esto ocurre «porque la realidad verdaderamente significativa para el hombre es tan solo la que él construye» (p. 17). El filósofo Dewey observa a comienzos del siglo xx cómo «la falta de comprensión de la naturaleza humana es causa primordial del menosprecio en que se la tiene, ya que cuando no se conoce íntimamente una cosa, siempre termina por despreciarla injustificadamente o por admirarla, sin que haya razón» (Dewey, 2014, p.15).

Para evitar que se convierta en una práctica 0 un interés transitorio es importarte que la educación se fundamente en una antropología acorde con la naturaleza del ser humano y no se guíe en exclusiva por las necesidades productivas y económicas. Es un valor intrínseco que integra la excelencia y la ética, que busca una «vida buena» para tener vidas plenas, éticas y productivas (Nucci, Krettenauer y Narváez, 2014). Así, Lackeus (2017) señala que la educación emprendedora que se orienta a los demás, que desarrolla en los alumnos la búsqueda del beneficio de los otros, mitiga las tendencias neoliberales que tiene esta disciplina.

El neoliberalismo en educación concibe a la persona en términos económicos, enfatiza la importancia del individuo autónomo y entiende la libertad como capacidad de elección (Lynch, 2006). Este planteamiento favorece el desarrollo de la inventiva y de la iniciativa humana, que están en la base de la iniciativa emprendedora, pero olvida que la libertad sirve al bien.

Las políticas educativas deben tener un objetivo intrínseco y perfectivo que posibilite la mejora integral de las capacidades de las personas en sí mismas. La labor educativa más importante es ayudar a las nuevas generaciones a encontrar valores que les ayuden a hacer y a decidir, que les den luz para dar respuesta a la pregunta ¿qué clase de vida quiero vivir y qué debo aprender para vivir esa vida? (Pring, 2016). En esta línea, la educación para el emprendimiento es una realidad doble, que acoge no solo valores económicos, sino que supone el desarrollo de la personalidad y el ejercicio de la libertad.

Por otro lado, es preciso señalar que la educación emprendedora tiene su origen en el ámbito económico y la educación 
empresarial está en el origen de la cultura emprendedora. Estos aspectos contribuyen a que tenga un marcado carácter económico y empresarial y que prevalezca la óptica que lo identifica con la generación de empresas (Pepin, 2012; Peña, Cárdenas, Rodríguez Martín y Sánchez-Lissen, 2015). En cierta manera es lógica esta confusión por la afinidad conceptual.

En buena parte de los países desarrollados y en vías de desarrollo, la educación para el emprendimiento se incluye en los planes de estudio desde edad temprana (Mahieu, 2006; Volkmann, Wilson, Mariotti, Rabuzzi, Vyakarnam y Sepulveda, 2009; Mwasalwiba, 2010; Draycott y Rae, 2011; Rosendahl, Sloof y Van Praag, 2014; Moberg, 2014; Jayawarna, Jones y Macpherson, 2015; Eurydice, 2016). Este paradigma se basa en la premisa de que, en una economía madura, el crecimiento en cantidad y calidad de la actividad empresarial es proporcional a la calidad de la formación empresarial. Sin embargo, esta opinión no es unánime. Algunos autores se oponen con disparidad de argumentos: se plantean dudas sobre su eficacia (Matlay, 2006, 2007); se cuestiona el valor educativo que supone aprender a desarrollar un plan de negocio (Neck y Greene, 2011; Jones y Penaluna, 2013) o se considera que promueve la ideología de mercado y el neoliberalismo por lo que encuentra oposición en buena parte del profesorado (Erkkilä, 2000; Lynch, 2006; Johannisson, 2010; Rae, 2010; Komulainen et al., 2011; Gill, 2014; Fougère et al., 2017; Lackéus, 2017). Algunos autores proponen que la educación para el emprendimiento en los ciclos de primaria apoye el desarrollo de las habilidades emprendedoras de los estudiantes pero con un enfoque no comercial (Iredale, 1993; Lewis y Massey, 2003; Hitty, 2008). Asimismo, se plantean algunas propuestas que sirven como incubadoras para desarrollar proyectos comunitarios y se impulsa una educación emprendedora desde un marcado cooperativismo. Se busca el impacto más allá de lo económico, la identificación de los problemas del entorno y la propuesta de soluciones creativas (Bel, Fernández-Guadaño, Lejarriaga y Martín-López, 2016).

Si la educación para el emprendimiento se ciñe al desarrollo del tejido empresarial, cabe el riesgo de que se convierta en una pedagogía de la empresarialidad (Bernal Guerrero, 2015). De acuerdo con Barroso (2015), una educación temprana en emprendimiento, carente de sentido educativo, cuestionaría la conveniencia de defender una educación temprana especializada en otras opciones profesionales 0 asociada a otras formas de vida.

Estas ideas hacen pensar en la necesidad de plantear una educación para el emprendimiento que priorice a la persona, colabore en el crecimiento de todas las dimensiones y no se limite al desarrollo de proyectos empresariales. Desde esta perspectiva, la propuesta que se desarrolla a continuación profundiza - dentro de los límites que este espacio ofrece- en el marco teórico de la educación para el emprendimiento. Este es un requisito previo que contribuye a consolidar la competencia emprendedora tanto como competencia transversal que desarrolla un enfoque educativo favorecedor del crecimiento del 
potencial emprendedor de los estudiantes, como cuando se plantea como asignatura independiente y con identidad propia dentro de los planes de estudios.

\section{Propuesta de fundamentación antropológica para la educación emprendedora}

Se parte de la hipótesis de que la educación emprendedora requiere de un fundamento conceptual de raíz antropológica que sitúe a la persona en el centro de la reflexión pedagógica, responda a un proyecto de ser humano y de sociedad acorde con la naturaleza humana y contribuya al crecimiento armónico de todas las dimensiones de la persona. Se ha elegido la óptica de la antropología humanista porque protege a la persona, evita que se prioricen como educativos intereses transitorios o que la educación se guíe por las necesidades productivas y económicas. Recientemente la UNESCO ha impulsado una concepción humanista de la educación y el desarrollo que se aleja del utilitarismo y el economicismo. Se plantea como deseable que la educación integre las dimensiones múltiples de la existencia humana, basada en sólidos fundamentos éticos y morales, y contribuya a forjar las dimensiones sociales, económicas y medioambientales de un nuevo modelo de desarrollo sostenible (UNESCO, 2015).

Del concepto que se tenga de persona humana se desprende la correspondiente perspectiva pedagógica. Es necesario escuchar las sugerencias que plantea la sociedad, pero la reflexión del educador se preocupa por proteger los fines y principios que rigen el quehacer educativo. Esta es una tarea dispositiva que lleva a elegir lo que se enseña de manera que se favorezca la formación humana y contribuya a la plenitud personal.

En esta línea resalta la importancia de considerar a la persona como principio y no solo como resultado (Altarejos y Naval, 2011). Está en juego la atribución de sus acciones libres y responsables no como mero resultado de factores sociales, económicos, biológicos o técnicos. Partiendo de este planteamiento, se ofrece una propuesta de fundamentación antropológica que, desde los presupuestos humanistas, profundice en la contribución de la educación emprendedora al crecimiento de todas las dimensiones de la persona. De manera directa y principal se contribuye al desarrollo de: 1) la dimensión intelectual, 2) la dimensión social y 3) la dimensión moral.

Este planteamiento resulta congruente con una educación «capaz de poner unidad en todos los posibles aspectos de la vida de un hombre» (García Hoz, 1981, p. 24). De esta manera, la educación para el emprendimiento se integra en el plan de estudios, de forma independiente o transversal, pero siempre como contribución al crecimiento de todas las dimensiones de la persona.

\subsection{Contribución al desarrollo de la di- mensión intelectual}

Entre todas las dimensiones del ser personal, destaca, en primer lugar, la intelectual, con una doble finalidad: la teórica, que proporciona un conocimiento del mundo, y la práctica, que ayuda a tratar con la 
realidad y descubre nuevas posibilidades. La educación para el emprendimiento se integra en la finalidad práctica, al contribuir al desarrollo de la creatividad que se entiende como mejora de la capacidad de pensar de manera diferente, además de la capacidad de resolución de problemas y el desarrollo del pensamiento estratégico, que permite la búsqueda de oportunidades y la disposición al logro.

En la actualidad, hay gran interés por la creatividad, potenciado por la valoración creciente de la innovación, a la que se considera herramienta básica para la sostenibilidad y para el desarrollo de cualquier proyecto. A su vez, la innovación interesa porque posibilita la mejora socio-económica y abre nuevas oportunidades empresariales (Comisión Europea, 2007; Wagner, 2014).

La creatividad, en cuanto capacidad de generar ideas y encontrar nuevas soluciones a los problemas, manifiesta la singularidad de la persona. Sus acciones muestran su modo singular de obrar, su carácter originario y creativo, dada la novedad que aporta cada quien en su irrepetibilidad. La creatividad encamina así a emprender acciones variadas como pensar, buscar relaciones, establecer objetivos y priorizar, dar respuesta a un medio ambiente cambiante, crear estrategias de futuro, diseñar o solucionar problemas de la vida (García Hoz, 1981; Pérez Alonso-Geta, 2009).

En consecuencia, la creatividad es importante para las personas y para la sociedad. Sin embargo, no se asocia en exclusiva con la generación de valor económico, sino que es un elemento de desarrollo personal. De otro modo, se deshumaniza. Es así como la educación emprendedora, anclándose en el carácter irrepetible del ser humano y de su libertad, lleva el sello de la intencionalidad constructiva que se orienta a la realización de acciones positivas, tanto para la sociedad como para las personas.

A lo largo del siglo xx, junto al concepto de creatividad se ha desarrollado el de innovación, que ha pasado a ocupar un lugar central en los escenarios de la política y de la economía mundial. Se han fortalecido los vínculos entre la creatividad, la innovación y el emprendimiento, como tres procesos interrelacionados en los que la capacidad de innovación genera valor y nuevas oportunidades (Edwards-Schachter, García-Granero, Sánchez-Barrioluengo, Quesada-Pineda y Amara, 2015).

El trinomio creatividad-innovación-emprendimiento se fundamenta en el modelo de creative destruction de Schumpeter $(2010)^{1}$. Este considera la creatividad como precursora del comportamiento innovador y característica central del potencial y del esfuerzo emprendedor de los individuos. Un comportamiento se considera emprendedor cuando existe innovación. Pero este, a su vez, debe ir acompañado de valores que promuevan una sociedad mejor (Ceberio, 2009).

\subsection{Contribución al desarrollo de la di- mensión social}

En segundo lugar, la educación para el emprendimiento contribuye al crecimiento de la dimensión social. En el presente 
trabajo se parte del enfoque de la sociología relacional (Donati, 2002, 2006). Su planteamiento es compatible con una educación que asienta sus raíces en la persona como ser que coexiste y que crece como ser donal (Polo, 2006). Ambas perspectivas, la sociológica y la antropológica, se complementan y resultan imprescindibles en los planes de estudio que prioricen la dimensión formativa y que al mismo tiempo atiendan a la relación con la realidad social.

La persona se realiza a nivel de esencia viviendo en sociedad no solo porque necesita relacionarse con los demás, sino porque es relación. Esta idea se vincula con el carácter sistémico humano, tal como la plantea Polo (2006), para el que sistémico supone la interrelación de elementos diversos, de modo que lo que incide en uno de ellos afecta de alguna manera a los otros.

Desde esta óptica, la educación para el emprendimiento contribuye, al mismo tiempo, al desarrollo personal y a la mejora y al cambio social. Su fundamento no solo reside en que la persona establece relaciones, sino en que es un ser relacional que se realiza mediante la donación, porque el ser humano no solo da, sino que se da cuando entrega sus dones (Altarejos y Naval, 2011). De esta manera, se proporciona una nueva visión a las relaciones estudiante-sociedad, en la que toda mejora en la persona se transforma de inmediato en una mejora para la sociedad.

La educación en clave relacional no se reduce a su utilidad, ni es un mero vehículo solucionador de problemas sociales. Entiende al hombre como sujeto principal de toda acción social, abierto a su entorno y a los demás, y en perenne compromiso con la sociedad y con la mejora social, más allá de su bienestar material. Busca educar ciudadanos activos y comprometidos, capaces de generar relaciones y bienes para los demás, acordes a la naturaleza humana y en los que la reciprocidad, el trabajo en equipo y la ayuda son elementos claves (Sandoval y Garro, 2012). Un reto de la educación para el emprendimiento es favorecer que se establezcan relaciones sociales sólidas, significativas y éticas y dar sentido a las mismas por encima de criterios de utilidad. Desde este planteamiento, los pilares que fundamentan la educación para el emprendimiento son las virtudes sociales como la sociabilidad, la solidaridad y la cooperación social, la dimensión de servicio y el bien común, porque muestran el potencial solidario y el carácter humanizador de las relaciones. Las personas son capaces de identificar objetivos y metas comunes en torno a necesidades compartidas y así, llegar a generar una identidad colectiva con la que cada uno de ellos se reconozca, siempre respetando y acogiendo la identidad personal de cada quien (Sandoval y Garro, 2012). De esta manera, se humanizan las relaciones y se evita el individualismo que niega el carácter relacional de la persona y la encierra en sí misma.

Siguiendo a Donati (2006) y Zamagni (2012) esto es posible si se prioriza a la persona y prima la relacionalidad de la reciprocidad, homo reciprocans, sobre la del intercambio, homo oeconomicus. Zamagni (2012) apela a una relacionalidad que no es la propia del intercambio, sino de la reciprocidad. La primera es instrumental: aunque 
se entre en relación con alguien, este no es más que un medio para obtener un fin. El principio de intercambio declara: te doy algo a condición de que tú me des a cambio el equivalente de su valor. Sin embargo, la reciprocidad no puede entenderse en términos de interés propio: te doy libremente algo para que tú puedas, a tu vez, dar a otros según tus capacidades.

Así, llevar a la práctica educativa el principio de reciprocidad se constituye en el objetivo práctico de la educación para el emprendimiento que se configura como medio de regeneración social y de preocupación por la persona. Este planteamiento otorga primacía a la relacionalidad de la reciprocidad, abandona la inmanencia y busca el bien común, no solo el intercambio de valor y el bienestar económico. Se aspira a suscitar la capacidad de detectar el bien y de llevarlo a la práctica, no solo de forma esporádica, sino como hábito estable.

\subsection{Contribución al desarrollo de la di- mensión moral}

Finalmente, la educación para el emprendimiento es, por su carácter práctico, ocasión y medio de educación moral. La calidad educativa está supeditada a la dignidad, profundidad y extensión de los valores que seamos capaces de suscitar (Díaz-Torres y Rodríguez-Gómez, 2008).

La educación moral se asocia inseparablemente con la educación intelectual. Aunque se diferencian en el concepto, no se separan de manera operativa. La perspectiva antropológica invita a que, desde el punto de vista educativo, la formación moral se centre en el crecimiento en virtudes (Peters, 1969; Altarejos y Naval, 2011; Nucci et al., 2014).

La crisis de las sociedades occidentales no se circunscribe a la productividad o a la economía, sino que hunde sus raíces en una crisis más profunda, antropológica y moral - tanto individual como social-, una crisis de convicciones y fundamentos que necesita fortalecer sus principios y fundamentar la tarea educativa de las políticas y de los sistemas de educación supranacionales. La educación emprendedora actúa como vínculo entre la sociedad y los individuos y es ocasión para desarrollar y mejorar la sociedad. Lo que se enseña, cómo se enseña y por qué se enseña influirá en la configuración de los sistemas políticos democráticos y en los valores de las generaciones futuras (Medina, 2001).

La educación para el emprendimiento fomenta el desarrollo de la sensibilidad moral de los estudiantes, para «construir de forma compartida principios morales con pretensión de universalidad sin dejar de propiciar condiciones que ayuden a reconocer las diferencias, los valores, las tradiciones y la cultura en general de cada comunidad» (Díaz-Torres y Rodríguez-Gómez, 2008, p. 163).

Son claros los vínculos de la educación emprendedora, por un lado, con la autonomía, porque desarrolla el conocimiento propio y el ejercicio responsable de la libertad; y, por otro, con el liderazgo, entendido en clave personal, donde el líder guía su propia vida según unos principios y sus competencias son hábitos que definen su identidad personal. 
El ejercicio de la autonomía tiene una especial importancia en las sociedades modernas, donde se vive un proceso de disolución de las responsabilidades (Bauman, 2013). A su vez, Arendt (1996) señala que la autonomía evita la tiranía de la mayoría y prepara para la tarea de renovar un mundo común. Según Altarejos y Naval (2011), el interés por la autonomía se vincula con el desarrollo de la libertad, que define a la naturaleza humana y supone un crecimiento de las posibilidades de acción, en la que el individuo colabora con la realización subjetiva del bien objetivo. En la actualidad, el desarrollo personal y la autorrealización individual tienen un fuerte peso en la tarea formativa. Suponen el desarrollo de la responsabilidad, además del ejercicio de la opinión propia y la toma de decisiones, el espíritu crítico, la asunción de riesgos y la integridad personal. El ejercicio de la autonomía estrecha sus relaciones con la responsabilidad. No se trata solo de una autonomía que lleva a realizar acciones, sino que, a través de ellas, se establece un compromiso con el mundo y se genera una identidad. Somos -en partefruto de lo que hacemos y a través de las propias acciones se asienta la condición humana.

A su vez, la educación para el emprendimiento tiene estrecha relación con el liderazgo, entendido en su dimensión personal. Se define como «la capacidad de guiar la propia vida según unos principios coherentes, con una recta comprensión del mundo de uno mismo, de modo que se consiga finalmente una vida lograda, es decir, una felicidad interior estable y profunda» (Cardona y García-Lombar- día, 2005, p. 137). Esta tarea es posible si se dota a la educación emprendedora de un fundamento antropológico que no varíe en función del contexto cultural y que haga duradero ese interés. Las competencias propias del liderazgo son hábitos que definen su identidad, dan sentido a la propia vida y despliegan su misión personal en el tiempo. Ese sentido de misión guía y promueve las competencias de liderazgo.

Desde la educación para el emprendimiento se puede contribuir al desarrollo de las virtudes cívicas, la solidaridad y la cooperación. Al mismo tiempo, estas fortalecen, desde el ámbito personal, el desarrollo de la responsabilidad personal y ayudan a valorar la amplitud de miras y los intereses morales. De esta manera, se constituyen en cimiento y contribuyen a la madurez moral. Juicio y carácter moral y cívico se aúnan. Este planteamiento se distancia de las propuestas que limitan la competencia emprendedora al desarrollo de habilidades técnicas o la generación de valor económico y empresarial. Se concibe la educación para el emprendimiento como ocasión de enriquecer la personalidad y forjar hábitos de responsabilidad y compromiso.

En la siguiente tabla se recoge, a modo de síntesis, la correspondencia entre las dimensiones de la persona y los indicadores más relevantes de la competencia emprendedora. Futuros trabajos nos permitirán ahondar en las estrategias metodológicas que implica su desarrollo entendido como oportunidad para el crecimiento de todas las dimensiones de la persona. 
TABLA 1. Dimensiones de la persona e indicadores de la competencia emprendedora.

\begin{tabular}{|l|l|l|}
\hline \multicolumn{1}{|c|}{ Dimensión intelectual } & \multicolumn{1}{c|}{ Dimensión social } & \multicolumn{1}{c|}{ Dimensión moral } \\
\hline Imaginación & Comunicación & Autonomía \\
\hline Creatividad & Sociabilidad & Responsabilidad \\
\hline Innovación & Servicio & Conocimiento propio \\
\hline Resolución de problemas & Trabajo en equipo & Toma de decisiones \\
\hline Búsqueda de oportunidades & Solidaridad & Capacidad crítica \\
\hline Iniciativa & Cooperación social & Pensamiento ético y sostenible \\
\hline Disposición al logro & & Liderazgo \\
\hline
\end{tabular}

Fuente: Elaboración propia.

\section{A modo de conclusión}

El interés por la educación para el emprendimiento se presenta como una tendencia educativa internacional que ha calado con fuerza en la institución educativa y se ha introducido en los planes de estudio de manera progresiva. Se fomenta su desarrollo como medio que propicie el cambio de mentalidad que necesita la sociedad para adecuarse a los requerimientos del siglo XXI, en los que la adaptación al cambio y el emprendimiento a lo largo de toda la vida se han convertido en un imperativo necesario. La crisis económica y las elevadas tasas de desempleo han incentivado su desarrollo.

El emprendimiento es un fenómeno social reciente pero es antiguo y universal en su dimensión humana. Lo que le ha hecho ser nuevo es que ha empezado a ser considerado como fenómeno económico (García del Dujo, 2015). Desde el punto de vista pedagógico, se detecta la falta de consenso acerca de las bases conceptuales que fundamentan el modelo educativo del emprendimiento. El espíritu emprendedor tiene su origen y su ámbito natural en el mundo empresarial (Peña et al., 2015). Su desarrollo corre paralelo a la teoría econó- mica y solo después se ha extendido su uso a otros ámbitos. Se trata, por tanto, de un fenómeno de raíz económica. A su vez, la aplicación práctica de esta materia es heterogénea; en gran parte de las ocasiones se limita al desarrollo de la capacidad productiva y económica, y se asocia con la mejora de las habilidades para crear empresas.

En este contexto, el punto de partida del presente artículo ha sido colaborar en la fundamentación teórica de la educación para el emprendimiento, de manera que contribuya a la mejora de la práctica educativa y se facilite la labor del profesorado (Pittaway, Aissaoui y Fox, 2017; Bernal Guerrero y Cárdenas, 2017; Lackéus, 2017).

Se adopta la perspectiva de la antropología humanista por dos motivos principales:

En primer lugar, porque la educación para el emprendimiento posee, como ha quedado de manifiesto, una fuerte identidad económica que se ve necesario contrarrestar. Esta tarea requiere situar a la persona en el centro de la reflexión pedagógica, responder a un proyecto de ser humano y de sociedad acorde con la natu- 
raleza humana y contribuir al crecimiento armónico de todas las dimensiones de la persona.

En segundo lugar, porque la tarea de emprender no se puede limitar a la mejora de habilidades empresariales, sino que, atendiendo a su origen, esta es una tarea específica del ser humano que no se limita a cubrir necesidades, sino que elige con libertad los medios para alcanzar un fin y trata de que lo que le rodea mejore con su aportación. La iniciativa, junto con la capacidad de cooperación, son los medios centrales de esta actividad.

Para finalizar, con este planteamiento de educación para el emprendimiento se desea, por un lado, dar respuesta a las necesidades personales del educando y contribuir a su desarrollo integral y, por otro, desarrollar la intención transformadora y potenciar el hábito de pensar y trabajar para mejorar el entorno. Con este doble prisma se favorece tanto la formación integral de los alumnos como el fortalecimiento de los lazos entre educación, persona y sociedad.

\section{Nota}

${ }^{1}$ La creation destruction (creación destructiva) es un concepto que introdujo Joseph Schumpeter en la economía. Es un proceso que favorece el crecimiento económico que se considera esencial dentro de la economía de mercado porque impulsa la evolución constante, el cambio y la reorganización productiva de la economía.

\section{Referencias bibliográficas}

Altarejos, F. y Naval, C. (2011). Filosofía de la educación. Pamplona: Universidad de Navarra.
Arendt, H. (1996). La crisis en la educación. Barcelona: Península.

Asamblea General de las Naciones Unidas (2000). Resolución A/55/L.2 del 13 de septiembre de 2000. Declaración del Milenio. Recuperado de http://xurl.es/40q1w (Consultado el 28-05-2019).

Asamblea General de las Naciones Unidas (2015). Resolución A/70/L.1 de 21 de octubre de 2015 Transformar nuestro mundo: la Agenda 2030 para el Desarrollo Sostenible. Recuperado de http://xurl.es/nevkr (Consultado el 28-05-2019).

Barrio, J. M. (2013). La innovación educativa pendiente. Formar personas. Barcelona: Erasmus.

Barroso, C. (2015). Emprendedor: cuando el término es más que una palabra. En L. Núñez-Cubero (Coord.), Cultura emprendedora y Educación (pp. 133-142). Sevilla: Universidad de Sevilla.

Bauman, Z. (2013). Sobre la educación en un mundo líquido. Barcelona: Paidós.

Becker, G. S. (1964). Human Capital. New York: Columbia University Press.

Bel, P., Fernández-Guadaño, J., Lejarriaga, G. y Martín-López, S. (2016). La iniciativa emprendedora como base para la creación de empresas de participación. Un instrumento para la innovación social. Cooperativismo y Desarrollo, 24 (108), 1-50.

Bernal Guerrero, A. (2015). Sobre la relevancia del factor personal en la investigación en educación emprendedora. En L. Núñez Cubero (Coord.), Cultura emprendedora y Educación (pp. 127-132). Sevilla: Universidad de Sevilla.

Bernal Guerrero, A., Granados, P. D. y González, M. D. (2014). Economy of knowledge, Entrepreneurial Culture and Employability in the field of Education. An Approximation to the Spanish Case. Procedia-Social and Behavioral Sciences, 139, 168-174.

Bernal Guerrero, A. y Cárdenas, A. (2017). Evaluación del potencial emprendedor en escolares. Una investigación longitudinal. Educación XX1, 20 (2), 73-94.

Business, Innovation, \& Skills Department (2015). Fulfilling our potential: Teaching excellence, social mobility and student choice. Recuperado de http://xurl.es/pjfpm (Consultado el 28-05-2019). Cardona, P. y García-Lombardía, P. (2005). Cómo desarrollar las competencias de liderazgo. Pamplona: Universidad de Navarra. 
Ceberio, I. (2009). Innovación y creatividad en las personas. En M.- J. Madariaga (Eds.), Filosofía de la innovación (pp. 169-177). Madrid: Plaza Janés.

Comisión Europea (2006). Entrepreneurship education in Europe: Fostering Entrepreneurial mindsets through education and learning. The Oslo Agenda for Entrepreneurship Education in Europe. Recuperado de http://xurl.es/7784t (Consultado el 28-05-2019).

Comisión Europea (2012). COM (2012) 669/final, del 20 de noviembre de 2012. Comunicación de la Comisión al Parlamento Europeo, al Consejo, al Comité Económico y Social Europeo y al Comité de las Regiones. Un nuevo concepto de educación: invertir en las competencias para lograr mejores resultados socioeconómicos. Recuperado de http://xurl.es/n1p74 (Consultado el 28-05-2019).

Comisión Europea (2014). COM (2014) 130 final, del 5 de marzo de 2014. Comunicación de la Comisión al Parlamento Europeo, al Consejo, al Comité Económico y Social Europeo y al Comité de las Regiones, Balance de la Estrategia Europa 2020 para un crecimiento inteligente, sostenible e integrador. Recuperado de http:// xurl.es/zvv5i (Consultado el 28-05-2019).

Consejo Europeo (2000). Conclusiones de la Presidencia. Consejo Europeo de Lisboa 23 y 24 de marzo 2000. Recuperado de http://xurl.es/ gunbm (Consultado el 28-05-2019).

Dewey, J. (2002). Human nature and conduct. New York: Dover Publication.

Dewey, J. (2014). Naturaleza humana y conducta. México: Fondo de Cultura Económica.

Díaz Torres, J. M. y Rodríguez Gómez, J. M. (2008). La educación en valores como estrategia de desarrollo y consolidación de la persona moral. Estudios sobre Educación, 15, 159-169.

Donati, P. (2002). Introduzione alla sociología relazionale. Milán: Franco Angeli.

Donati, P. (2006). Repensar la sociedad. El enfoque relacional. Madrid: Ediciones Internacionales Universitarias.

Draycott, M. y Rae, D. (2011). Enterprise education in schools and the role of competency frameworks. International Journal of Entrepreneurial Behaviour \& Research, 17 (2), 127-145.
Edwards-Schachter M., García-Granero, A., Sánchez-Barrioluengo, M., Quesada-Pineda H. y Amara N. (2015). Disentangling competences: Interrelationships on creativity, innovation and entrepreneurship. Thinking skills and creativity, 16, 27-39.

Erkkilä, K. (2000). Entrepreneurial Education. Mapping the debates in the United States, the United Kingdom and Finland. New York: Garland Publishing.

Espejo, B. (2001). Políticas educativas para el nuevo siglo. Salamanca: Hespérides.

Eurydice (2016). La educación para el Emprendimiento en los centros educativos en Europa. Informe de Eurydice. Luxemburgo: Oficina de Publicaciones de la Unión Europea.

Fougère, M., Segercrantz, B. y Seeck, H. (2017). A critical reading of the European Union's social innovation policy discourse: (Re)legitimizing neoliberalism. Organization, 24 (6), 819-843.

García del Dujo, A. (2015). De la utilidad de las virtudes clásicas (para resguardar al sujeto de las inclemencias del tiempo). En L. Núñez Cubero (Coord.), Cultura emprendedora y Educación (pp. 61-68). Sevilla: Universidad de Sevilla.

García Hoz, V. (1981). Educación personalizada. Madrid: Rialp.

Gilead, T. (2012). Education and the Logic of Economic Progress. Journal of Philosophy of Education, 46 (1), 113-131.

Gill, R. (2014). If you're struggling to survive dayto-day': class optimism and contradiction in entrepreneurial discourse. Organization, 21 (1), 50-67.

Hitty, U. (2008). Enterprise education in different cultural settings and at different school levels. En A. Fayolle y P. Kyrö (Eds.), The dynamics between entrepreneurship, environment and education (pp. 131-148). Cheltenham-Northampton: Edward Elgar Publishing.

Hornsby, J. S., Messersmith, J., Rutherford, M. y Simmons, S. (2018). Entrepreneurship everywhere: across campus, across communities and across borders. Journal of Small Business Management, 56 (1), pp. 4-10.

Ibáñez-Martín, J. A. y Fuentes, J. L. (Eds.) (2017). Educación y capacidades. Hacia un nuevo enfoque del desarrollo humano. Madrid: Dykinson. 
Iredale, N. (1993). Enterprise education in primary schools: a survey in two northern LEAs'. Education + Training, 35 (4), 22-30.

Jayawarna, D., Jones, O. y Macpherson, A. (2015). Becoming an entrepreneur: the unexplored role of childhood and adolescent human capital. En D. Rae y C. L. Wang (Eds.), Entrepreneurial Learning: New Perspectives in Research, Education and Practice (pp. 45-72). London: Routledge.

Johannisson, B. (2010). The agony of the Swedish school when confronted by entrepreneurship. En K. Kogen y J. Sjovoll (Eds.), Creativity and Innovation. Preconditions for entrepreneurial education (pp 91-106). Trondheim: Tapir Academic Press.

Jones, C. y Penaluna, A. (2013). Moving beyond the business plan in enterprise education. Education + Training, 5 (8/9), 804-814.

Jones, P., Maas, G. y Pittaway, L. (2017). New perspectives on entrepreneurship education. En Entrepreneurship Education: New Perspectives on Entrepreneurship Education (pp. 1-13). Bingley: Emerald Publishing.

Komulainen, K., Naskali, P., Korhonen, M. y Keskitalo-Foley, S. (2011). Internal entrepreneurship -a Trojan horse of the neoliberal governance of education? Finnish pre-and in-service teachers' implementation of and resistance towards entrepreneurship education. Journal for Critical Education Policy Studies, 9 (1), 341-374.

Lackéus, M. (2017). Does entrepreneurial education trigger more or less neoliberalism in education? Education + Training, 59 (6), pp. 635-650.

Ley Orgánica 2/2006, de 3 de mayo, de educación (LOE). Boletín Oficial del Estado, 106, 2006, de 4 de mayo de 2006, páginas 17158 a 17207. Recuperado de https://www.boe.es/eli/es/ lo/2006/05/03/2 (Consultado el 04-07-2019).

Ley Orgánica 8/2013, de 9 de diciembre, para la mejora de la calidad educativa (LOMCE). Boletín Oficial del Estado, 295, de 10 de diciembre de 2013, páginas 97858 a 97921 . Recuperado de https://www.boe.es/buscar/doc.php?id=BOEA-2013-12886 (Consultado el 04-07-2019).
Ley Orgánica 14/2013, de 27 de septiembre, de apoyo a los emprendedores y su internacionalización. Boletín Oficial del Estado, 233, de 28 de septiembre de 2013, páginas 78787 a 78882 . Recuperado de https:/www.boe.es/diario_boe/txt.php?id=BOE-A-2013-10074 (Consultado el 04-07-2019).

Lewis, K. y C. Massey (2003). Delivering enterprise education in New Zealand. Education + Training, 45 (4), 197-206.

Lynch, K. (2006). Neo-liberalism and marketization: the implications for higher education. European Educational Research Journal, 5 (1), 1-17.

Mahieu, R. (2006). Agents of change and policies of scale: a policy study of entrepreneurship and enterprise in education (Tesis doctoral). Umeå Universitet, Umeå, Suecia.

Martínez Clares, P. y González Morga, N. (2018). Las competencias transversales en la universidad: propiedades psicométricas de un cuestionario. Educación XX1, 21 (1), 231-262.

Matlay, H. (2006). Entrepreneurship education: more questions than answers? Education + Training, 48 (5), 293-295.

Matlay, H. (2007). Pertinent questions in entrepreneurship education: why, what, when, and how? Education + Training, 49 (8/9), 279-302.

Medina, J. R. (2001). Sistemas contemporáneos de educación moral. Madrid: Ariel.

Michavila, F., Martínez, J. M., Martín-González, M., García-Peñalvo, F. J. y Cruz-Benito, J. (2016). Barómetro de empleabilidad y empleo de los universitarios en España, 2015. Madrid: Observatorio de Empleabilidad y Empleo Universitario.

Mincer, J. (1974) Schooling, Experience and Earnings. Cambridge, MA: The National Bureau of Economic Research.

Moberg, K. (2014). Two approaches to entrepreneurship education: The different effects of education for and through entrepreneurship at the lower secondary level. The International Journal of Management Education, 12 (3), 512-528.

Mwasalwiba, S. (2010). Entrepreneurship education: a review of its objectives, teaching methods, and impact indicators. Education + Training, 52 (1), 20-47. 
Nabi, G., Liñán, F., Fayolle, A., Krueger, N. y Walmsley, A. (2017). The impact of entrepreneurship education in higher education: A systematic review and research agenda. Academy of Management Learning and Education, 16 (2), 277-299.

Neck, H. M. y Greene, P. G. (2011). Entrepreneurship Education: Known Worlds and New Frontiers. Journal of Small Business Management, 49 (1), 55-70.

Nucci, L., Krettenauer, T. y Narváez, D. (Eds.) (2014). Handbook of moral and character education. New York: Routledge.

Orden ECD/65/2015, de 21 de enero, por la que se describen las relaciones entre las competencias, los contenidos y los criterios de evaluación de la educación primaria, la educación secundaria obligatoria y el bachillerato. Boletín Oficial del Estado, 25, de 29 de enero 2015, páginas 6986 a 7003. Recuperado de https://www.boe. es/buscar/doc.php?id=BOE-A-2015-738 (Consultado el 05-07-2019).

Peña, J. V., Cárdenas, A., Rodríguez Martín, A. y Sánchez-Lissen, E. (2015). La cultura emprendedora como objetivo educativo: marco general y estado de la cuestión. En L. Núñez Cubero (Coord.), Cultura emprendedora y Educación (pp. 19-60). Sevilla: Universidad de Sevilla.

Pepin, M. (2012). Enterprise education: a Deweyan perspective. Education + Training, 54 (8/9), 801-812.

Pérez Alonso-Geta, P. M. (2009). Creatividad e innovación: una destreza adquirible. Teoría de la Educación, 21 (1), 179-198.

Peters, R. S. (1969). El concepto de educación. Buenos Aires: Paidós.

Pittaway, L., Aissaoui, R. y Fox, J. (2017). Social Constructionism and Entrepreneurial Opportunity. En M. Karatas-Ozkan, A. Fayolle, S. Ramoglou, K. Nicolopoulou (Eds.), Philosophical Reflexivity in Entrepreneurship (pp. 1-32). New York: Routledge.

Polo, L. (2006). Ayudar a crecer. Cuestiones filosóficas de la educación. Pamplona: Universidad de Navarra.

Pring, R. (2016). Una filosofía de la educación políticamente incómoda. Madrid: Narcea.
Rae, D. (2010). Universities and enterprise education: responding to the challenges of the new era. Journal of Small Business and Enterprise Development, 1 (4), 591-606.

Ruiz-Herrero, J. A. (julio de 2013). Nuevos espacios productivos en el capitalismo cognitivo: el ejemplo del emprendimiento. XI Congreso Español de Sociología. Simposio llevado a cabo en la conferencia de la Federación Española de Sociología, Madrid.

Sandoval, L. Y. y Garro, N. (2012). La sociología relacional: una propuesta de fundamentación sociológica para la institución educativa. $E d u$ cación y Educadores, 15 (2), 247-262.

Schultz, T. (1961) Investment in Human Capital. The American Economic Review, 51 (1), 1-17.

Schumpeter, J. (2010). Capitalism, Socialism and Democracy. Abingdon: Routledge Classics.

Organización de las Naciones Unidas para la Educación, la Ciencia y la Cultura (2015). Replantear la educación ìhacia un bien común mundial? París: UNESCO. Recuperado de https:// unesdoc.unesco.org/ark:/48223/pf0000232697 (Consultado el 05-07-2019).

Volkmann, C., Wilson, K. E., Mariotti, S., Rabuzzi, D., Vyakarnam, S. y Sepulveda, A. (2009). Educating the next wave of entrepreneurs-unlocking entrepreneurial capabilities to meet the global challenges of the 21st Century. A Report of the Global Education Initiative. Ginebra: Word Economic Forum.

Wagner, T. (2014). Creando innovadores. La formación de los jóvenes que cambiarán el mundo. Madrid: Kolima.

Zamagni, S. (2012). Por una economía del bien común. Madrid: Ciudad Nueva.

\section{Biografía de las autoras}

Arantxa Azqueta es Doctora en Ciencias de la Educación por la Universidad de Navarra, con una tesis sobre educación para el emprendimiento calificada con sobresaliente y mención cum laude. En la actualidad, es Profesora Asociada de la Universidad de Navarra y Profesora en el Departamento de Teoría e Historia de la 
Educación, en la Facultad de Educación de la Universidad Internacional de La Rioja (UNIR).

(iD https://orcid.org/0000-0003-2514-5989

Concepción Naval es Catedrática de Universidad y Decana en la Facultad de Educación y Psicología de la Universidad de Navarra. Ha sido Oliver Smithies Lecturer en la Oxford University; Visiting Professor en el Teachers College en la Columbia University y Visiting Scholar Research en la Universidad de Harvard, en la Universidad de Notre Dame (EEUU): Oficina Internacional de la Educación (UNESCO), en el St. Edmund's College de la Cambridge University y en la Boston University.

iD http://orcid.org/0000-0002-5927-9398 